\title{
RESEARCH
}

Open Access

\section{Hybrid WGWO: whale grey wolf optimization-based novel energy-efficient clustering for EH-WSNs}

\author{
Rajkumar Singh Rathore ${ }^{1}$, Suman Sangwan ${ }^{1}$, Shiv Prakash ${ }^{2}$, Kabita Adhikari $^{3}$, Rupak Kharel ${ }^{4^{*}}$ (D) and Yue Cao
}

\author{
* Correspondence: r.kharel@mmu.ac. \\ uk \\ ${ }^{4}$ Department of Computing and \\ Mathematics, Faculty of Science and \\ Engineering, Manchester \\ Metropolitan University, Manchester \\ M1 5GD, UK \\ Full list of author information is \\ available at the end of the article
}

\begin{abstract}
The energy harvesting methods enable WSNs nodes to last potentially forever with the help of energy harvesting subsystems for continuously providing energy, and storing it for future use. The energy harvesting techniques can use various potential sources of energy, such as solar, wind, mechanical, and variations in temperature. Energy-constrained sensor nodes are small in size. Therefore, some mechanisms are required to reduce energy consumption and consequently to improve the network lifetime. The clustering mechanism is used for energy efficiency in WSNs. In the clustering mechanism, the group of sensor nodes forms the clusters. The performance of the clustering process depends on various factors such as the optimal number of clusters formation and the process of cluster head selection. In this paper, we propose a hybrid whale and grey wolf optimization (WGWO)-based clustering mechanism for energy harvesting wireless sensor networks (EH-WSNs). In the proposed research, we use two meta-heuristic algorithms, namely, whale and grey wolf to increase the effectiveness of the clustering mechanism. The exploitation and exploration capabilities of the proposed hybrid WGWO approach are much higher than the traditional various existing metaheuristic algorithms during the evaluation of the algorithm. This hybrid approach gives the best results. The proposed hybrid whale grey wolf optimization-based clustering mechanism consists of cluster formation and dynamically cluster head $(\mathrm{CH})$ selection. The performance of the proposed scheme is compared with existing state-of-art routing protocols.
\end{abstract}

Keywords: Clustering, Cluster head (CH), WGWO, Whale optimization algorithm (WOA), Grey wolf optimization (GWO)

\section{Introduction}

Recently, lots of research are going on various aspects of the energy harvesting wireless sensor networks (EH-WSNs) [1]. EH-WSNs are playing a crucial role in multiple applications [2, 3], and also, in EH-WSNs, sensor nodes (SNs) can harvest energy from the environment. The energy harvested SNs can improve their sensing range and lifetime as compared to conventional SNs, which are depending on battery power for their operations. Energy harvesting methods help the SNs to get the permanent power supply from the sources available in the environment. Moreover, this scheme raises two

(c) The Author(s). 2020 Open Access This article is licensed under a Creative Commons Attribution 4.0 International License, which permits use, sharing, adaptation, distribution and reproduction in any medium or format, as long as you give appropriate credit to the original author(s) and the source, provide a link to the Creative Commons licence, and indicate if changes were made. The images or other third party material in this article are included in the article's Creative Commons licence, unless indicated otherwise in a credit line to the material. If material is not included in the article's Creative Commons licence and your intended use is not permitted by statutory regulation or exceeds the permitted use, you will need to obtain permission directly from the copyright holder. To view a copy of this licence, visit http://creativecommons.org/licenses/by/4.0/. 
concerns, such as the availability of energy resources and the amount of energy collected. For successfully deploying WSNs, two issues that need attention are energy harvesting methods, and second is energy management issues [4]. In energy harvesting schemes, environmental energy is converted into electrical energy. The energy management mechanism consists of lowering energy consumption. The overall aim is to maximize the remaining network energy.

In EH-WSNs, we can achieve a reliable and energy-efficient packet delivery by enhancing the existing protocols. And for this, the scheme should provide adequate forwarding opportunities during the route-finding phase to find a robust route. Along this route, packets greedily proceed to the destination through node's participation [5]. In EH-WSNs, it is a fact that SNs have a limitation in terms of energy and communication ability. Therefore, there is a need to design a routing scheme for EH-WSNs to improve data delivery to the receiver. The solution is energy-balanced routing schemes based on the characteristics of forwarder nodes. The next forwarder is selected as per the quality of the link and forwarder remaining energy [6].

Sensor nodes should have a higher energy harvesting rate as compared to their energy consumption rate. But, this condition is not usually meet. The duty cycle (DC) method should be used to achieve this condition [7]. By increasing the network connectivity (i.e., increasing the node's sensing range) in the wireless communication systems, the convergence rate enhances. But, this increases energy consumption and consequently decreasing the lifetime of EH-WSNs [8]. Reducing energy consumption and, therefore, extending the network lifetime is a critical parameter for the successful deployment of WSNs. There are various schemes developed for achieving the above objective, such as multi-hop schemes, cooperative transmission schemes, and dutycycle schemes [9]. The main limitation of wireless charging is the risk of electromagnetic exposure in WSNs. Also, wireless chargers can deplete the battery. The energy harvesting technique has low risk. Harvesting energy from the environment can be used effectively to energize the SNs. A power management scheme is needed for harvesting the energy from the environment for efficiently managing the energy of sensors, and this mechanism results in maximizing the sensors' duty cycle [10].

For designing robust EH-WSNs, the mobility concept is used to avoid communication hindrances due to unstable energy variations. The mobile collector concept is getting attention recently. In this technique, based on the sensed data, energy consumption is balanced in the network. Now, researchers are working on designing distributed algorithms to increase the network performance by changing the data rates that adapt to the unstable environmental energy fluctuations [11]. In EH-WSNs, data transmission with QoS constraints is a significant research challenge. Various research work utilizes multipath routing to achieve reliability in EH-WSNs. However, multipath routing has high energy costs. We can improve the efficiency of QoS routing in EHWSNs by the appropriate selection of the forwarding candidate set [12].

There is an urgent need in EH-WSNs to answer two simple questions on how to correctly estimate the energy consumption and selection of energy-efficient routes to send the sensed data to the base station (BS). The formulation of a heuristic energy-efficient data sensing and routing scheme is needed in EH-WSNs to answer the above questions [13]. There are a large number of different energy-efficient routing schemes in EHWSNs that addresses the issue of lowering energy consumption. A new energy 
harvesting technology is getting the attention that uses piezoelectric nanogenerators to supply power to the nano sensor; these are the sensors which cannot be charged by traditional energy harvesters [14]. In a high dense WSNs, there is a redundancy of data during sensing tasks. The reason is apparent; for precise and accurate monitoring, a large number of sensor nodes are utilized. Hence, in this case, there is a need for data fusion and aggregation for saving energy. In this way, total communication cost and energy consumption improve due to a reduction in the size and number of messages exchanged among SNs [15]. It is a well-known fact that in an EH-WSNs scenario, transmission distance affects energy consumption as well as harvested energy at SN. Energy consumption is directly proportional to the square of the distance, and harvested energy is inversely proportional. There must be some mechanism to pick the nodes which are near to the path to help in the smooth transmission of data in the network [16]. Furthermore, the protocols should consider the different energy levels of the receiver, which is lacking in currently available protocols for EH-WSNs. Therefore, there is a need for the medium access control (MAC) protocol for QoS in EH-WSNs. Further, the protocol should also adjust the receiver DC according to its current energy level [17].

Several kinds of research are going on to lower energy consumption by using clustering-based schemes in EH-WSNs. The hybrid approach consisting of static and dynamic clustering is a new method. The hybrid approach utilizes two parameters, such as distributed-centralized schemes and multi-hop routing. Also, this scheme considers the criteria, such as the level of energy, estimation of harvested energy, and the number of neighbors [18]. Apart from two vibrant issues of EH-WSNs, such as reducing the energy expenditure and enhancing the network lifetime, the other important aspect in EH-WSNs is to improve its operating performance also. To improve the working performance of EH-WSNs, the BS can form unequal size clusters, and select cluster heads (CHs) correspondingly, in which the clusters close to the BS have a smaller size. Then, the BS builds efficient paths among each CHs [19]. Next, EH-WSNs need a cross-layer protocol that can perform load-balancing adaptively, consisting of geographic-based routing, and can select relay nodes (RNs) efficiently [20].

The size of the sensor nodes (SNs) is small, and they have limited energy capabilities. With limited energy capabilities, they are having constrained in the communication process. Therefore, some advanced methods are needed that are capable of reducing energy consumption and enhancing the network lifetime in EH-WSNs. Clustering is a prominent energy-efficient technique. Clustering balances the energy consumption among all SNs and minimizes traffic and overhead during data transmission phases of WSNs.

The clustering-based approach consists of CHs selection, and SNs send data to the nearest $\mathrm{CH}$. CHs undertake data aggregation and compression. More responsibilities to $\mathrm{CHs}$ will require more energy consumption to process and transmit each cluster's data, which will result in premature and irregular network depletion. There is not a general approach to determine and optimize the dissipated energy and network lifetime longevity. Existing various methods focus on improving one feature and may increase energy consumption in other. Also, existing approaches do not consider the quantitative determination of energy consumption for the complete network.

For further development, this paper have proposed the hybrid WGWO (whale and grey wolf optimization)-based novel energy-efficient clustering for EH-WSNs. 
The significant insights and offerings of this article can be listed as follows.

- We propose the hybrid whale grey wolf optimization (WGWO) algorithm for the optimal number of clusters formation as well as dynamically selecting the cluster heads $(\mathrm{CHs})$. The proposed approach shows the novel research, where the proposed WGWO optimization algorithm selects the energy-efficient clusters with efficient cluster heads (CHs) in a dynamic fashion. We design the hybrid whale grey wolf optimization (WGWO) algorithm by the integration of two novel optimization methods, such as the whale optimization algorithm and the grey wolf optimization algorithms.

- Further, the relay nodes (RNs) are selected based on energy efficiency criteria, and later they are prioritized.

- Finally, the proposed WGWO technique is compared with existing methods such as enhanced threshold sensitive stable election protocol (ETSSEP) [21], link-aware clustering mechanism (LCM) [22], tree-based clustering (TBC) [23], and partitionbased low-energy adaptive clustering hierarchy (LEACH) [24] in terms of performance measuring attributes, namely, delay, packet delivery ratio (PDR), energy consumption, and network lifetime and throughput. The numerical analysis appraises that the average percentage improvements of the proposed scheme outplay the existing methods when compared.

The research article is organized and summarized as per following. Section 2 depicts the related works. Section 3 describes the proposed scheme. Section 4 demonstrates performance analysis. Finally, Section 5 concludes the research article.

\section{Related works}

\subsection{Energy consumption minimization strategies in EH-WSNs}

Zahedi et al. [25] propose a swarm intelligence-based forwarding (SIF); SIF uses the efficient clustering algorithm to form the clusters, and then appropriate $\mathrm{CHs}$ are selected. This technique generates a balanced as well as the precise number of clusters. The main objective of this routing scheme is to enhance the network's lifetime, and it can be applied to a wide variety of applications. Next, Zhang et al. [26] propose an efficient routing scheme. The basis of this scheme is an efficient clustering technique, as well as a topology control mechanism. The clustering technique is utilized to find out the optimal number of clusters.

Further, in the topology control mechanism, sensor nodes (SNs) find out the efficient path, based on the optimal forwarding communication area. The main characteristic of this protocol is to minimize energy consumption and enhance the lifetime of the network. Further, Darabakh et al. [27] propose a novel clustering and routing scheme in EH-WSNs. This scheme aims to lower energy consumption by utilizing the multi-layer architecture. This scheme reduces the communication range, which also decreases the communication overhead. Furthermore, this paper proposes a multi-hop routing algorithm, which considers the two factors, namely, total distance and the energy of RNs.

\subsection{Efficient connectivity scheme in EH-WSNs}

Mohemed et al. [28] present the solution of the routing hole issue due to energy depletion in EH-WSNs. The paper effectively resolves the issue of the premature ending of 
the network lifetime, where the BS is far away. The proposed scheme maintains connectivity in an energy-efficient manner. In EH-WSNs, the degree of sensor nodes (SNs) affects the performance of the network. Therefore, we need to drive the perfect node degree with reduced consumption of energy. Furthermore, Mohamed et al. [29] propose a routing mechanism with minimum transmission energy requirement. The proposed scheme, with an optimal node degree, performs effective data monitoring tasks in WSNs.

\subsection{Concept of minimum cost route, geographic routing, and multi-hop graph-based routing in EH-WSNs}

In WSNs, the efficient energy management of the SNs is a big challenge. Therefore, special attention is deeply required. In high range data transmission, multi-hop routings are used. The multi-hop routing scheme has the limitation of finding efficient routes. Fawzy et al. [30] propose an energy-efficient scheme that is based on the Dijkstra algorithm. Higher energy nodes are selected to work as relays. The Dijkstra algorithm aims to search for the minimum cost route efficiently.

Further, Huang et al. [31] propose an efficient geographic routing scheme. The proposed scheme gives enhanced route recovery from a routing hole issue. It dynamically uses the three factors to make routing decisions, such as information related to location, residual energy computation, and total energy consumption. Earlier developed geographic routing schemes use only local location information to route data. Therefore, conventional geographic routing approaches suffer from the routing hole problem. Hence, existing protocols suffer from longer delivery delays and the lower delivery ratio.

Furthermore, Rhim et al. [32] propose a multi-hop graph-based technique for efficient routing schemes in WSNs, which aims to enhance the network lifetime. This technique consists of two phases such as the clustering phase and routing phase. The clustering phase consists of the formation of clusters and the further selection of $\mathrm{CHs}$. The routing phase is dedicated to dynamically building the multi-hop routes between $\mathrm{CHs}$ and the sink.

\subsection{Concept of probabilistic routing schemes, relay transmission probability, and role of opportunistic routing in EH-WSNs}

Hawbani et al. [33] primarily focus on probabilistic routing schemes. Probabilistic routing can be of two types, fixed and adaptive. Fixed schemes are having a fixed probability of forwarding. But in the adaptive case, various parameters are considered, such as distance, traffic, and energy to determine the probability of forwarding. They provide the conclusion that the optimal probability of forwarding is not universal, and it depends on many topological parameters such as density, location, and transmission range. Next, in EH-WSNs, the two issues are still challenging due to the fluctuating and unpredictable nature of harvested energy such as how to optimize energy efficiency and designing dynamic routing protocols. Li et al. [34] propose an efficient routing scheme in EH-WSNs by considering the relay transmission probability and residual energy. This scheme aims to achieve balanced routing with enhanced transmission characteristics. Further, the performance of WSNs can be significantly increased with the help of an opportunistic routing (OR) scheme. Traditional routing schemes use the pre- 
determined routes for packets transmission. OR scheme uses the metric for the selection of candidates as forwarders. Now in case of OR, the waiting issue is solved. But a large number of candidates affect the packet delivery. Hawbani et al. [35] propose a scheme that combines two main parts. First is defining the candidate's zone by each node. Second, the candidates are prioritized by using the OR metric.

\subsection{Recent developments in routing schemes for EH-WSNs}

Mottaghinia et al. [36] propose a routing scheme that considers three factors such as fuzzy-logic concept, distance, and energy-aware routing (FLDEAR). It is known as the FLDEAR protocol. This protocol uses two fuzzy inference systems in routing. The main feature of this protocol is the efficient use of buffer management. Next, Tang et al. [37] propose an efficient bionic hybrid preferable routing protocol (EHPRP) for EH-WSNs. It is also known as EHPRP. This scheme aims to optimally use the harvested energy to improve the lifespan of the network. Further, this approach would prominently minimize the delay in processing and save energy. Also, Lu et al. [13] propose a routing scheme that considers three steps primarily first, estimating the link quality; second, optimally allocating the energy for data monitoring and routing; and third, sending the collected data to the base station along energy-efficient routes. It is known as energyefficient data sender routes strategies (EEDSRS).

\subsection{Comparison of clustering protocols}

In the partition-based LEACH scheme [24], the complete network is divided into various sectors, and one of the sensor nodes ( $\mathrm{SNs}$ ) is designated as a $\mathrm{CH}$, which is having higher energy. In this approach, the attempt is made for enhancing the network's lifetime, but unbalanced consumption of energy is the prominent issue that arises here due to poor path selection.

In the tree-based clustering approach [23], the data delivery ratio improves inside the clusters only. The problem of unbalanced consumption of energy also exists in this approach. Also, in this approach, the packet delivery ratio is poor in the transmission of processed data from cluster head to base station due to adopting the single-hop transmission scheme.

Link-aware clustering mechanism (LCM) [22] utilizes the link quality attribute for routing. This approach is having higher communication overhead; also, excess energy is used for re-clustering, which occurs at regular intervals.

In enhanced threshold sensitive stable election protocol (ETSSEP) [21], the probability function is utilized for the election of $\mathrm{CH}$; the probability function is dynamic. Due to poor route selection strategy, this approach suffers from a network reliability issue.

Table 1 shows the features of some clustering protocols briefly.

From the above literature, it can be concluded that clustering is a necessary means to minimize energy consumption and prolong network life.

\section{Proposed hybrid WGWO: whale grey wolf optimization-based novel energy-efficient clustering in EH-WSNs}

WSNs are constrained in terms of energy efficiency and communication capabilities. Most of the research on WSNs has mainly concentrated on the design of energy and 
Table 1 Merits and demerits of various clustering protocols in WSNs

\begin{tabular}{|c|c|c|}
\hline Clustering protocols & Merits & Demerits \\
\hline Partition-based LEACH [24] & Improvement in network lifetime. & Unbalanced energy consumption. \\
\hline $\begin{array}{l}\text { Tree-Based Clustering (TBC) } \\
\text { [23] }\end{array}$ & $\begin{array}{l}\text { Improvement in packet delivery but only } \\
\text { inside the clusters. }\end{array}$ & $\begin{array}{l}\text { Energy consumption is uneven, } \\
\text { higher delay. }\end{array}$ \\
\hline $\begin{array}{l}\text { Link-aware clustering } \\
\text { mechanism (LCM) [22] }\end{array}$ & $\begin{array}{l}\text { Reliable routes are discovered by } \\
\text { considering link quality. Enhancement in } \\
\text { network lifetime. }\end{array}$ & $\begin{array}{l}\text { Communication overhead increases, } \\
\text { excess energy consumption for re- } \\
\text { clustering. }\end{array}$ \\
\hline $\begin{array}{l}\text { Enhanced threshold sensitive } \\
\text { stable election protocol } \\
\text { (ETSSEP) [21] }\end{array}$ & Improvement in network lifetime. & $\begin{array}{l}\text { Reduction in packet delivery and } \\
\text { network reliability performance. }\end{array}$ \\
\hline
\end{tabular}

computationally efficient algorithms and protocols. EH-WSNs can provide continuous and controllable energy for enabling the dead sensors to resume operation. The other important aspect of EH-WSNs is to improve its operating performance also. For enhancing the working performance of EH-WSNs, the BS can form unequal size clusters, and select $\mathrm{CHs}$ correspondingly, in which the clusters close to the BS have a smaller size. Then, the BS builds efficient paths among each CHs. Next, EH-WSNs need a crosslayer protocol that can perform load-balancing adaptively, consisting of geographicbased routing, and it should select relay nodes efficiently.

Clustering methods have various significant advantages. Clustering provides energy efficiency, scalability to decrease the energy utilization of SNs in EH-WSNs. Clustering reduces network overhead and traffic. Clustering prevents redundant messages from being exchanged during the communication. In clustering, $\mathrm{CHs}$ perform data compression and data aggregation task, which results in reduced energy consumption in the network. Also, the transmission range among SNs reduces in clustering mechanism. In the clustering mechanism, only $\mathrm{CHs}$ are having high-range data transmission. In the clustering mechanism, $\mathrm{CHs}$ play a major role, and therefore their selection is one of the most critical problems in the clustering process. The traditional optimization algorithms are not capable enough to provide the appropriate solution in a specific time. Meta-heuristic methods are mainly used for these optimization problems to provide the best solution. Next, Fig. 1 demonstrates the clustering mechanism in detail.

\subsection{Objective function}

There are seven objective functions [38, 39]. These seven objective functions are covering all important aspects needed for energy-efficient clustering such as saving the energy by minimizing the number of $\mathrm{CHs}$, finding the current energy ratio, enhancing the link quality in clusters, minimizing the distance between $\mathrm{CHs}$ and $\mathrm{BS}$, reducing the intra-cluster distances, maximizing the inter-cluster distance between $\mathrm{CHs}$, and finally balancing the load between CHs. The proposed hybrid WGWO algorithm provides the best solution by minimizing all seven objective function. The fitness function for this is the sum of all seven objective functions with seven weight constants. The fitness function, which is defined in the proposed method, is described as follows:

$$
\mathrm{FF}=\operatorname{minimum}\left(m_{1} y_{1}+m_{2} y_{2}+m_{3} y_{3}+m_{4} y_{4}+m_{5} y_{5}+m_{6} y_{6}+m_{7} y_{7}\right)
$$

where $m_{1}, m_{2}, m_{3}, m_{4}, m_{5}, m_{6}$, and $m_{7}$ are weight constants defined by the user, and FF is the fitness function. Also, $y_{1}, y_{2}, y_{3}, y_{4}, y_{5}, y_{6}$, and $y_{7}$ are the objective functions. 


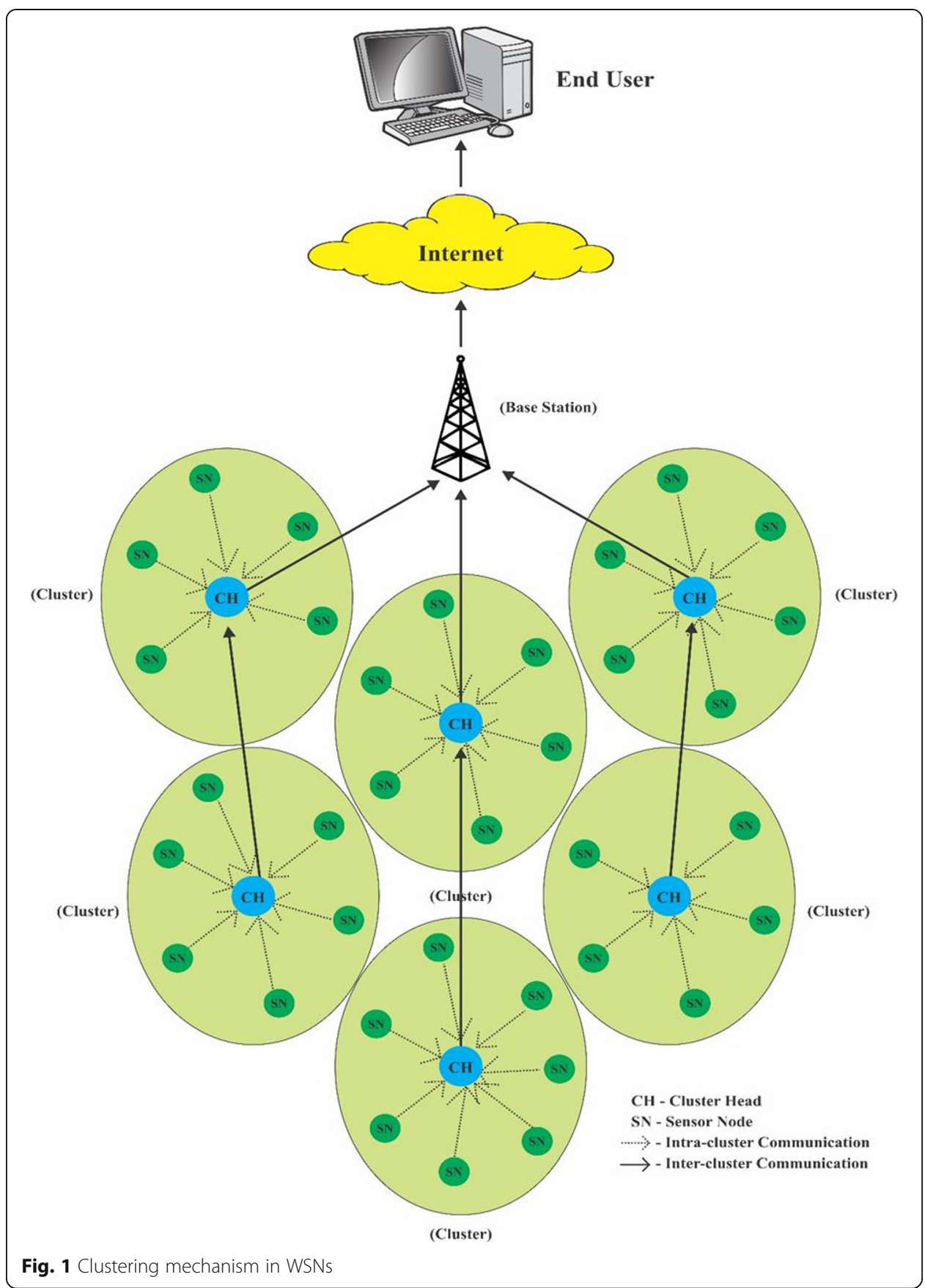

The proposed hybrid WGWO (whale grey wolf optimization) is used to find the best solution according to the fitness function. In the proposed hybrid algorithm, the solution is given by each agent. The best solution for the above-defined fitness function consists of the minimum number of clusters with high link quality and dynamically selected $\mathrm{CHs}$ with high remaining energy.

The first function is dedicated to save energy, and we know that if we can minimize the optimal number of $\mathrm{CHs}$, then energy consumption will be minimized. Equation (2) describes this clearly. 


$$
y_{1}=\frac{\text { optimal number of clusters }(c)}{\text { size of set of } \mathrm{CH} \text { contestants }(s)}
$$

The second function provides information about the current energy ratio; if there are total $M$ nodes, $R$ clusters then the second function is the ratio of the initial energy of nodes and the current $\mathrm{CHs}$ energy. Equation (3) describes this clearly.

$$
y_{2}=\frac{\sum_{p=1}^{M} \text { Energy }\left(\text { node }_{p}\right)}{\sum_{q=1}^{R} \text { Energy }\left(\text { cluster_head }_{q}\right)}
$$

The third function aims to improve the link quality in clusters. This function generates the clusters in such a way that distances between SNs are minimum. In the third function, Euclidean distance is calculated between the SN and the $\mathrm{CH}$. Equation (4) describes this clearly.



The fourth function minimizes the distance between $\mathrm{CHs}$ and BS. Here, the area is assumed to be $A \times A$; total clusters are $R$. Equation (5) describes it in detail.

$$
y_{4}=\frac{\frac{1}{R} \sum_{q=1}^{R} \text { euclidean_distance (cluster_head } q \text {, base_station) }}{\frac{A}{2}}
$$

The fifth function aims to reduce the intra-cluster distances of SNs and their corresponding CHs. The binary parameter is having value 1 if a particular node is assigned to a specific $\mathrm{CH}$; otherwise, its value is 0 . Equation (6) illustrates it in detail.

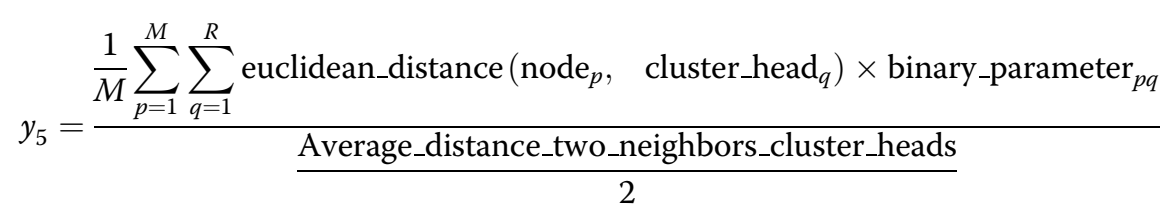

The sixth function is dedicated to maximizing the inter-cluster distance between CHs. Equation (7) represents it. $\left|C N_{q}\right|$ is the number of nodes in cluster $q$.

$$
y_{6}=\frac{\text { Average_distance_two_neighbors_cluster_heads }}{\frac{1}{\left|C N_{q}\right|} \sum_{q=1}^{\left|C N_{q}\right|} \text { euclidean_distance }(\text { cluster_head } q, \quad \text { cluster_head } r)}
$$

The seventh function is used for balancing the load between CHs. Equation (8) will reduce the maximum load between $\mathrm{CHs}$. $\left|C N_{q}\right|$ is the number of nodes in cluster $q$. Here, the area is assumed to be $A \times A$; total clusters are $R$. 


$$
y_{7}=\frac{\operatorname{MAXIMUM}\left(\left|C N_{q}\right|\right)}{\frac{1}{R} \sum_{q=1}^{R}\left(\left|C N_{q}\right|\right)}
$$

\subsubsection{Whale optimization algorithm (WOA)}

The mathematical modeling of WO A[40] is described in the below sections.

$$
\begin{aligned}
& \vec{U}=\left|\vec{H} \cdot \vec{N}^{*}(l)-\vec{N}(l)\right| \\
& \vec{N}(l+1)=\vec{N}^{*}(l)-\vec{Y} \cdot \vec{U}
\end{aligned}
$$

where "l" represents a current iteration, $\vec{Y}, \vec{H}$ represent coefficient vectors, $\vec{N}^{*}$ demonstrates the best solution, and $\vec{N}$ depicts a current state.

$$
\begin{aligned}
& \vec{Y}=2 \vec{y} \cdot \vec{i}-\vec{y} \\
& \vec{H}=2 \cdot \vec{i}
\end{aligned}
$$

where $\vec{y}$ reducing from 2 to 0 also $\vec{i} \in[0,1]$.

$$
\vec{N}(l+1)=\vec{U}_{\text {dist }} \cdot \exp ^{\operatorname{logarithmic}_{\text {spiral }} \cdot \cos } \quad(2 \Pi v)+\vec{N} *(l)
$$

where $\vec{U}_{\text {dist }}=\left|\vec{N}_{q}^{*}(l)-\vec{N}(l)\right|$

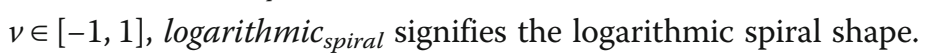

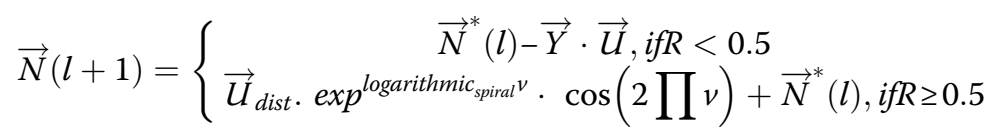

where $R \in[0,1]$.

$$
\begin{aligned}
& \vec{U}=\left|\vec{H} \cdot \vec{N}^{\text {random }}-\vec{N}\right| \\
& \vec{N}(l+1)=\vec{N}^{\text {random }}-\vec{Y} \cdot \vec{U}
\end{aligned}
$$

where $\vec{N}^{\text {random }}$ represents the current population random position vector.

\subsubsection{Grey wolf optimization (GWO) algorithm}

In GWO [41], alpha $(\alpha)$, beta $(\beta)$, delta $(\delta)$ wolves direct the tracking.

In GWO, the most important inspiration is to encircle a prey by guidance through $\alpha$, $\beta$, and $\delta$, which systematically establishes as beneath:

$$
\begin{aligned}
& Z(l+1)=Z(l)-B \cdot S \\
& S=\left|F \cdot Z_{p p}(l)-Z(l)\right|
\end{aligned}
$$

Here, $\mathrm{Z}$ represents the grey wolf position,

$Z_{p p}$ is the prey position,

$\mathrm{B}, \mathrm{F}$ are the coefficient vectors, and the number of iteration is defined by "l." 


$$
\begin{aligned}
& B=2 b \cdot d_{1}-b \\
& F=2 \cdot d_{2}
\end{aligned}
$$

where " $\mathrm{b}$ " decreases from 2 to 0 , and $\mathrm{d}_{1}$ and $\mathrm{d}_{2}$ are the random vectors from $[0,1]$. The parameter " $\mathrm{b}$ " is updated in every iteration within the range from 2 to 0 as below,

$$
b=2-l\left(\frac{2}{L}\right)
$$

At this point, "L" denotes the total number of iterations allowed.

The update procedure of wolves position takes place as per the following:

$$
\begin{aligned}
& Z_{1}=Z_{\alpha}(l)-B_{1} \cdot S_{\alpha} \\
& Z_{2}=Z_{\beta}(l)-B_{2} \cdot S_{\beta} \\
& Z_{3}=Z_{\delta}(l)-B_{3} \cdot S_{\delta}
\end{aligned}
$$

where $\mathrm{S}_{\alpha}, \mathrm{S}_{\beta}$, and $\mathrm{S}_{\delta}$ are obtained as follows:

$$
\begin{aligned}
& S_{\alpha}=\left|F_{1} \cdot Z_{\alpha}-Z\right| \\
& S_{\beta}=\left|F_{2} \cdot Z_{\beta}-Z\right| \\
& S_{\delta}=\left|F_{3} \cdot Z_{\delta}-Z\right|
\end{aligned}
$$

We can determine the solution for the next iteration as follows:

$$
Z(l+1)=\frac{\left(Z_{1}+Z_{2}+Z_{3}\right)}{3}
$$

The process of updating the wolf positions stopped when the maximum iteration achieved.

Next, Fig. 2 demonstrates the flow chart of the hybrid whale grey wolf optimization algorithm.

We have designed the hybrid scheme by integrating the whale and the grey wolf algorithms, which are known as novel approaches for optimization. Here, both algorithms provide the optimal best solutions simultaneously in each round of iteration; the best solution is selected in each round of iteration after comparing the solutions provided by both algorithms. Further this best solution is given to both algorithms to generate the optimal solutions which is much better than the past optimal solutions, and the cycle is repeated until maximum iterations.

A broad range of optimization problems has been solved by swarm intelligence and population-based algorithms. There are some complicated problems in which the traditional approaches cannot find the best solution at a particular time. WOA and GWO are the most recent meta-heuristic optimization algorithms.

Currently, meta-heuristic search algorithms which are based on swarm intelligence and population-based are used to provide the best solution in the form of energyefficient clustering methods in WSNs. For multidimensional search space, traditional algorithms are not able to give solutions in a specific time, and in these circumstances, meta-heuristic optimization algorithms provide an appropriate solution. 


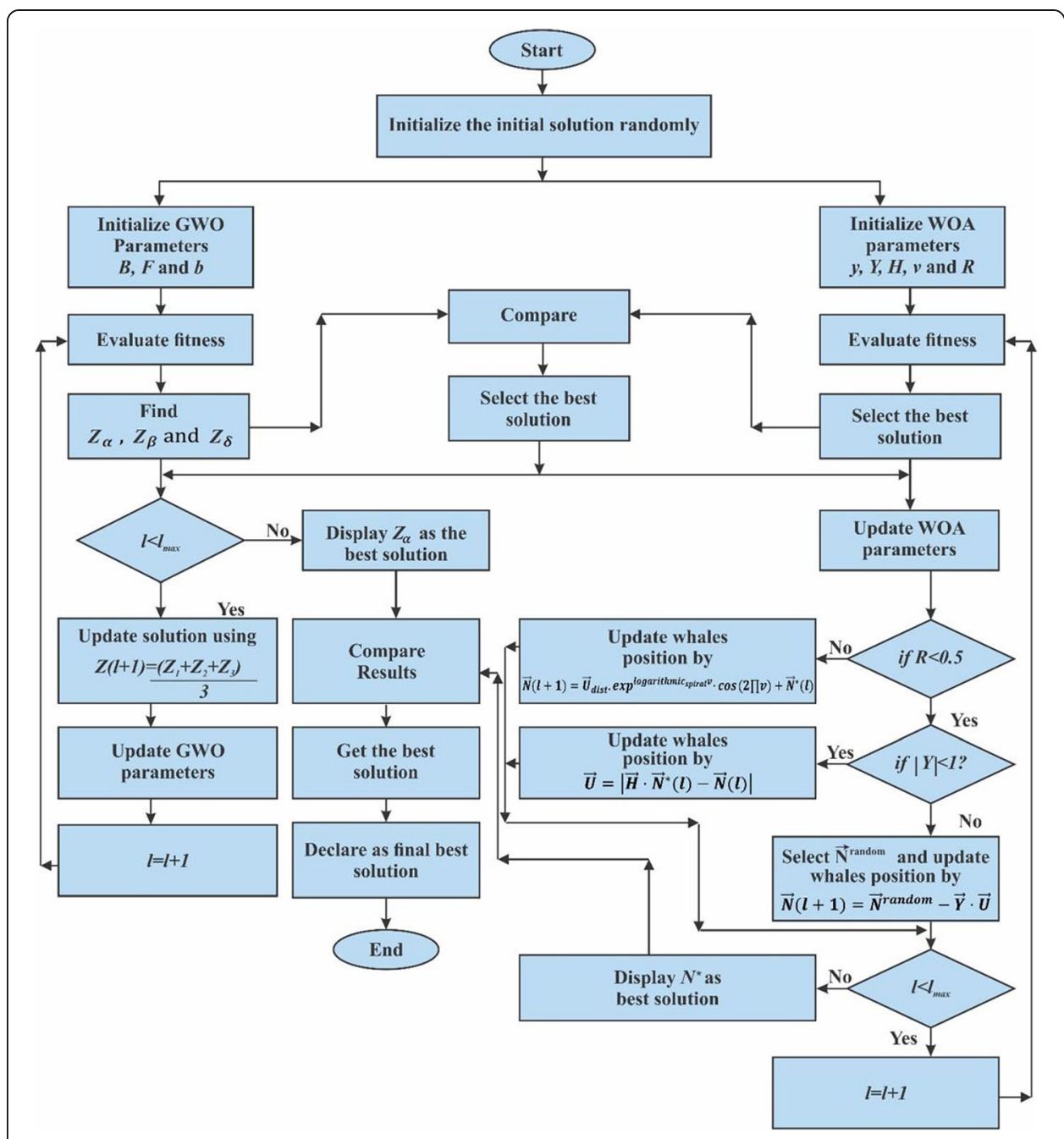

Fig. 2. Flow chart of the hybrid whale grey wolf optimization algorithm (WGWO)

\subsection{Relay nodes selection and prioritization scheme}

Relay nodes (RNs) are initially selected by considering three energy focused attributes, namely, energy-consumption [42, 43], congestion rate (CR) $[44,45]$, and also EDCW[46] metric.

The energy consumption parameter consists of energy utilized in data transmission, reception, and also energy utilized in data fusion.

$$
\operatorname{Energy}_{\text {transmission }}(R, m)=\text { Energy }_{\text {circuit_loss_transmission }}+\text { Energy }_{\text {circuit_loss_amplifier }}
$$

Here, $R$ is the range for $m$ bits data transmission, Energy circuit_loss_transmission $_{\text {depicts }}$ energy consumption in circuit, and Energy $y_{\text {circuit_loss_amplifier }}$ illustrates the energy consumption in the amplifier.

Equation (30) represents the energy consumption in receiving $m$ bits of data.

$$
\text { Energy }_{\text {receiving_data }}(m)=m \times \text { Energy }_{\text {transmission_per_unit_bit }}
$$

Similarly, Eq. (31) exhibits energy utilization in fusing $m$ bit data. 


$$
\text { Energy }_{\text {fusing }}(m)=m \times \text { Energy }_{\text {fusing_unit_bit_data }}
$$

The congestion rate parameter is utilized for evaluating the relay nodes (RNs) load.

$$
\text { Congestion } \_ \text {Rate }_{h}=\frac{\sum_{h=1}^{Q} \sum_{\text {node }_{h} \in \text { route }_{h k}^{p}} C D\left(\text { node }_{h}\right)-\sum_{\text {node }_{h} \in \text { route }_{h k}^{m}} C D\left(\text { node }_{h}\right)}{\sum_{h=1}^{Q} \sum_{\text {node }_{h} \in \text { route }_{h k}^{p}} C D\left(\text { node }_{i}\right)}
$$

Here, the degree of connectivity is represented by $C D\left(\right.$ node $\left._{i}\right)$, and route ${ }_{h k}$ is used for the route.

In the EDCW [46] metric, the RNs set is formulated by utilizing the information about the neighbors of the SNs. The EDCW of particular node $e_{i}$ can be expressed as given in Eq. (33). It is the sum of single-hop and multihop transmission.

$$
\text { EDCW }\left(\text { node }_{h}\right)=\frac{1}{\sum_{k \in F S(h)} L P(h, k)}+\frac{\sum_{k \in F S(h)} L P(h, k) \cdot E D C W(k)}{\sum_{k \in F S(h)} P(h, k)}+\text { weight }
$$

Here, weight represents the cost of forwarding. Also, $L P(h, k)$ is the success probabilities, and $F S(h)$ is the set of forwarders.

Next, we calculate the expected operating transmission cost (EOTC) [47] and prioritize the RNs by using EOTC. Therefore, the actual relay list is built for each node.

The EOTC of single-hop data forwarding, $\operatorname{EOTC}\left(T_{\text {single-hop }}^{u v}(w)\right)$ at particular slot $w$ is derived from the sum of transmission cost and the wait cost as given in Eq. (34).

$$
\operatorname{EOTC}\left(T_{\text {single-hop }}^{u v}(w)\right)=\operatorname{cost}\left(T_{\text {wait }}^{u v}(w)\right)+\operatorname{cost}\left(T_{\text {transmission }}^{u v}(w)\right)
$$

The multi-hop method of data transmission uses the EOTC metric in single-hop and average EOTC metric values of all subsequent RNs. The below Eq. (35) model this concept.

$$
\operatorname{EOTC}\left(T^{u v}(w)\right)=\operatorname{EOTC}\left(T_{\text {single-hop }}^{u v}(w)\right)+\frac{\sum_{r \in \operatorname{Set}_{\text {intial }}^{v}} \operatorname{EOTC}\left(T^{v r}(w)\right)}{\operatorname{size}\left(\operatorname{Set}_{\text {initial }}^{v}\right)}
$$

where size $\left(\operatorname{Set}_{\text {initial }}^{\nu}\right)$ depicts the relay set, and $\operatorname{EOTC}\left(T_{\text {single-hop }}^{u v}(w)\right)$ is the $\operatorname{EOTC}$ of receiver node $v$ for a single hop in the RN set. Next, EOTC is used for the prioritization of the relay nodes. We can form the actual list of RNs for each node based on EOTC by using the previous relay set. The RNs having lower EOTC values will get a higher priority.

\section{Results and discussions}

In this section, the network simulator (NS2) is utilized for implementing the hybrid WGWO based novel energy-efficient clustering mechanism for EH-WSNs. The sensor nodes (SNs) are varied from number 100 to 500. Table 2 represents the simulation 
Table 2 The simulation parameter and its value used for analysis

\begin{tabular}{ll}
\hline Parameter & Value \\
\hline Area sizex & $450 \mathrm{~m} \times 450 \mathrm{~m}$ \\
MAC protocol & $802 \_11$ \\
Antenna & Omnidirectional \\
Packet size & $128 \mathrm{bytes}$ \\
Transmission energy per node & $09 \times 10^{-3} \mathrm{~J}$ \\
Receiving energy per node & $2.9 \times 10^{-3} \mathrm{~J}$ \\
The initial energy of each sensor node & $0.7 \mathrm{~J}$ \\
Simulation time & $650 \mathrm{~s}$ \\
\hline
\end{tabular}

parameter and its value. The comparative analysis is carried out in the proposed research by comparing the proposed WGWO scheme with the schemes, namely, ETSSEP, LCM, TBC, and partition-based LEACH. The various performance parameters taken into consideration are energy consumption, network lifetime, throughput, packet delivery ratio (PDR), and delay.

Figure 3 shows the performance of the proposed WGWO scheme for delay parameter as compared with existing schemes. After analyzing the graph, it is clear that the proposed approach is having a lower delay than the existing schemes.

The delay parameter of the WGWO scheme is minimum due to the reason that we have used the multi-objective functions for energy-efficient clusters formation and dynamically selecting the cluster heads. Further, we have used the hybrid metaheuristic algorithms for the best solution in the form of optimal clusters formation and dynamically cluster heads selection; furthermore, in our approach, RNs are selected based on

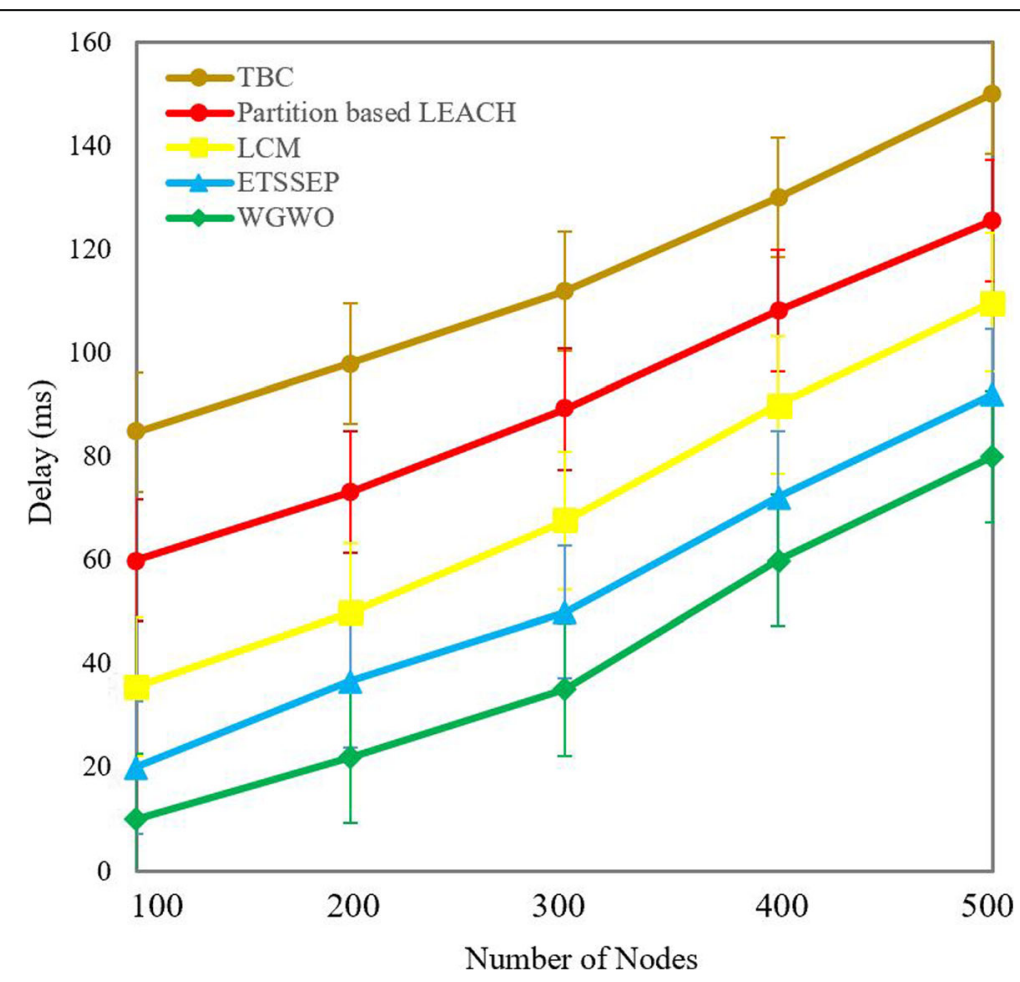

Fig. 3 Delay (ms) vs. variation in the number of nodes 
energy-efficient parameters, and later RNs are prioritized for efficiently transmitting the packets through optimal route without route breakages. Therefore, the probability of re data forwarding is also very low in our proposed approach. Hence, total time spent is low in communication mechanism, and this validates our claim of lower delay. Also, Table 3 shows the comparative analysis of WGWO for the delay parameter.

The average percentage improvements of WGWO in terms of delay are 29.99, 52.38, 58.98, and 67 as compared to ETSSEP, LCM, partition-based LEACH, and TBC, respectively. Detailed analysis of the delay parameter for WGWO is depicted in Fig. 4.

Figure 4 consists of five different columns. Initially, these five different columns represent the value of delay parameter on 100, 200, 300, 400, and 500 nodes, and later in this figure these five different columns represent the percentage improvements, and finally, sixth columns represent the average percentage improvements comparing with each scheme, respectively.

Figure 5 shows the performance of the proposed WGWO scheme for the packet delivery ratio (PDR) parameter as compared with existing schemes. After inspecting the graph, we can conclude that the proposed WGWO approach has a maximum PDR.

The PDR parameter of our proposed WGWO scheme is higher due to the reason that packet drop and control overhead is reduced in our proposed scheme; also, in our proposed approach delay is less for sending the packets from source to destination. Energy-efficient parameters are used for RNs selection. Therefore, incompetent RNs are avoided during routing. Also, in our proposed approach reliability is high in selecting the next forwarders as compared with existing schemes. Hence, the packet delivery ratio is maximum in our proposed approach, and accordingly network performance is improved. Next, Table 4 shows the comparative analysis of WGWO for the packet delivery ratio parameter.

The average percentage improvements of WGWO in terms of packet delivery ratio are $10.47,25.62,40.65$, and 55.78 as compared to ETSSEP, LCM, partition-based $\mathrm{LEACH}$, and TBC, respectively. Detailed analysis of the packet delivery ratio parameter for WGWO is depicted in Fig. 6.

Again, Fig. 6 consists of five different columns, and initially, these five different columns represent the value of PDR parameter on 100, 200, 300, 400, and 500 nodes; also, later in this figure these five different columns represent the percentage improvements, and finally, sixth columns represent the average percentage improvements comparing with each scheme, respectively.

Figure 7 exhibits the performance of the proposed WGWO scheme for the throughput parameter as compared with existing schemes. After observing the graph, it is clear that the proposed approach is having a higher throughput than the existing schemes.

Throughput depends on various factors such as delay, packet drop, and control overhead. The proposed scheme has less packet drop, control overhead, and delay. Therefore, throughput is higher in our scheme. Also, in our proposed approach reliable and optimal path is selected for data transmission using energy-efficient multiple parameters such as energy consumption, congestion rate, and EDCW metric. The probability of selecting the inefficient nodes in data routing is high in existing schemes, and consequently, throughput is less. In our proposed scheme, next forwarders are selected efficiently with a shorter range of communication and less energy consumption. Table 5 shows the comparative analysis of WGWO for the throughput parameter. 


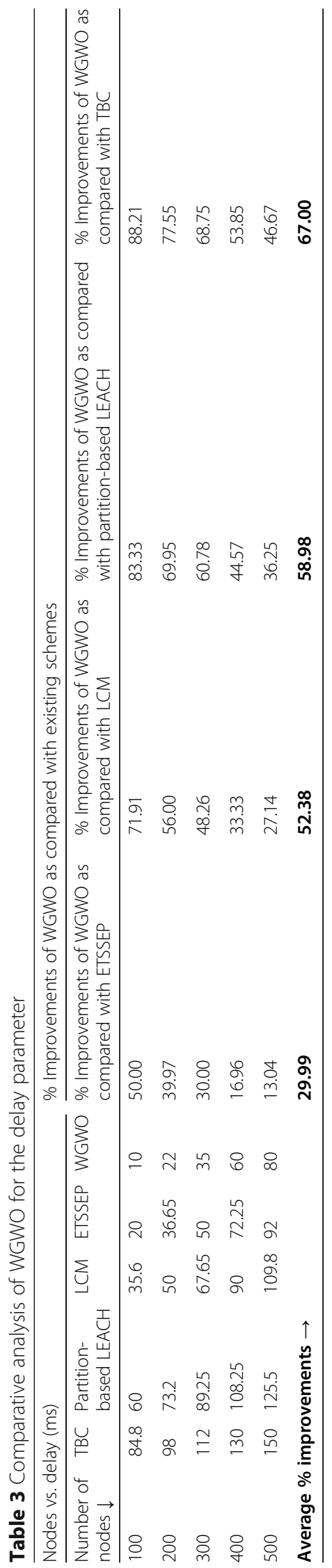




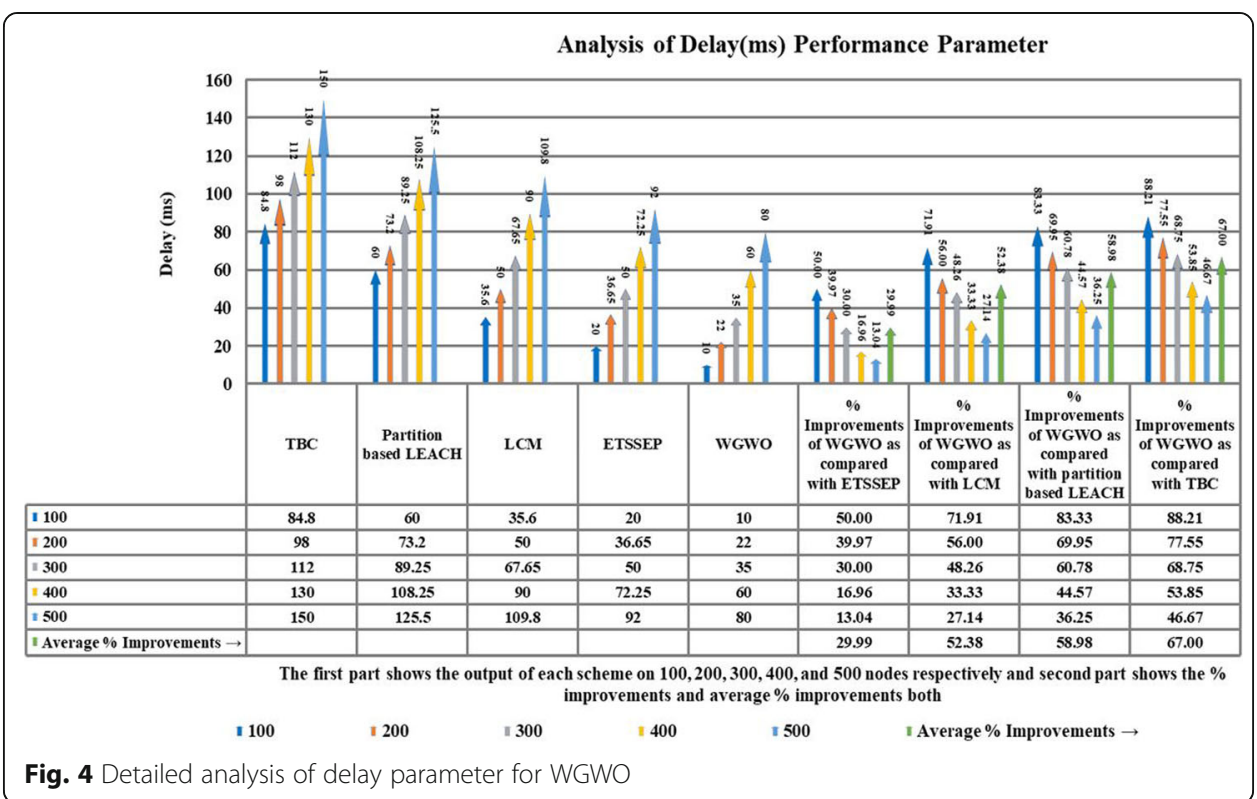

The average percentage improvements of WGWO in terms of throughput are 11.57, 26.73, 40.75, and 53.62 as compared to ETSSEP, LCM, partition-based LEACH, and $\mathrm{TBC}$, respectively. Detailed analysis of the throughput parameter for WGWO is depicted in Fig. 8.

Once again, Fig. 8 consists of five different columns. Same as the previous figure, these five different columns represent the value of throughput parameter on 100, 200,

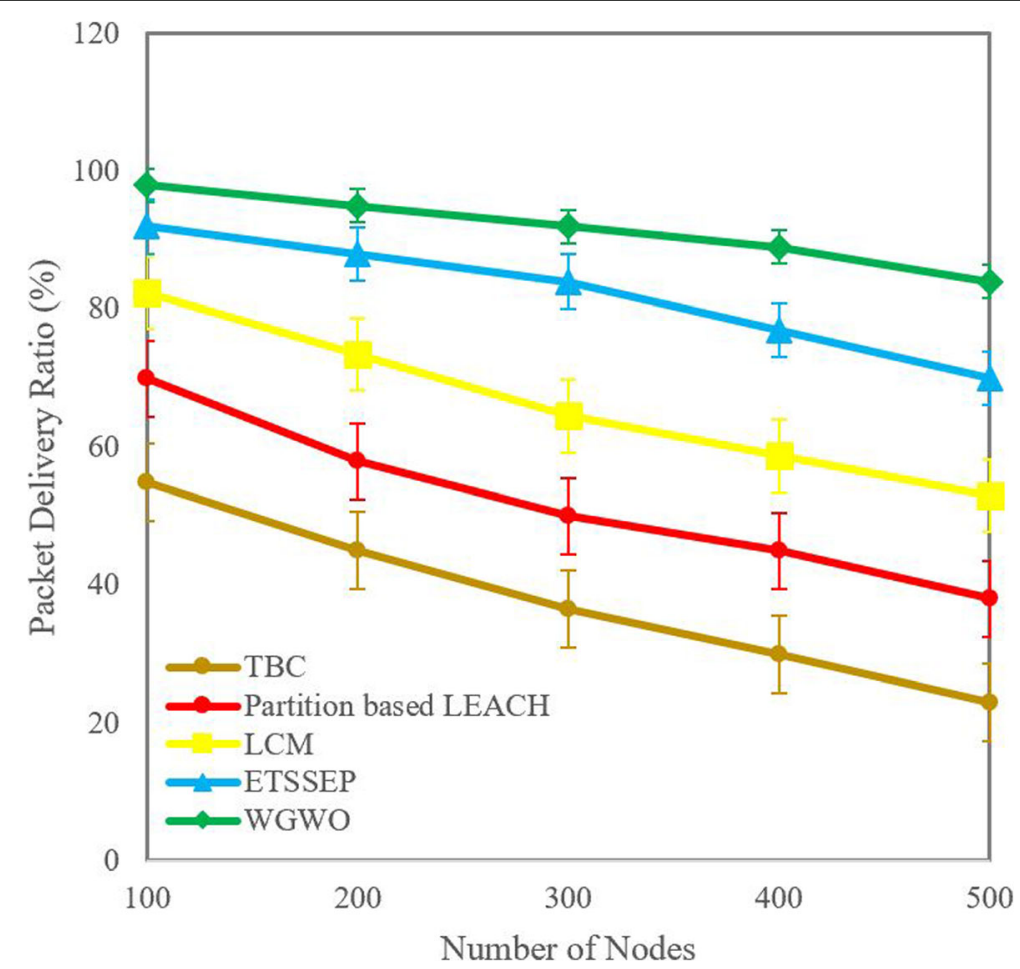

Fig. 5 Packet delivery ratio (PDR) (\%) vs. variation in the nodes 


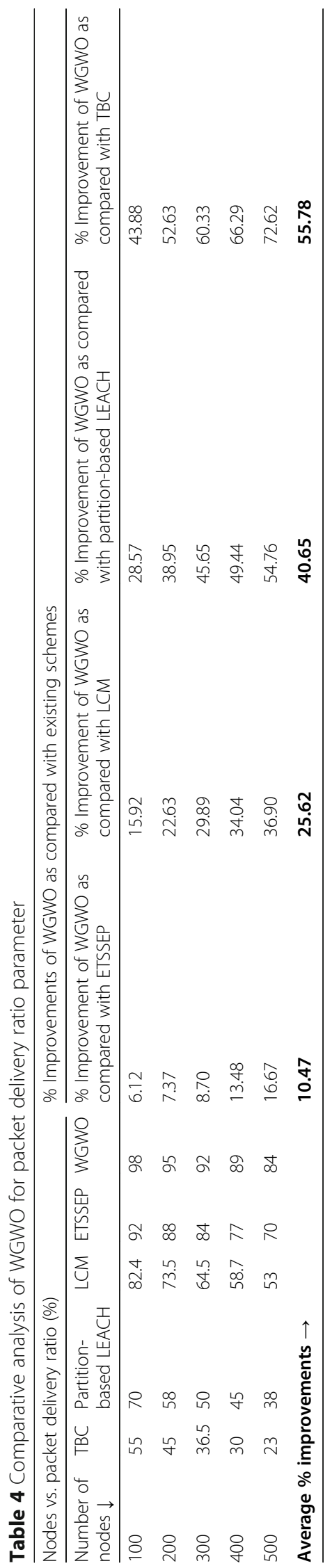




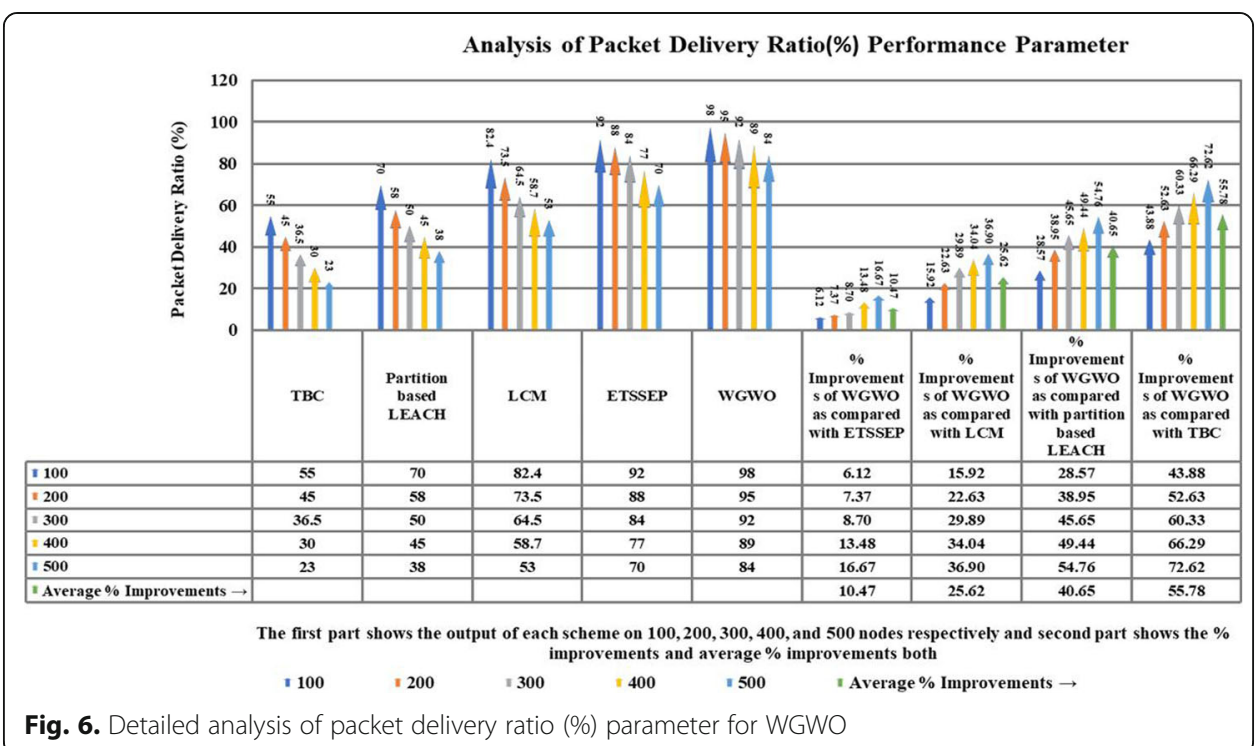

300, 400, and 500 nodes, and later in this figure, these five different columns represent the percentage improvements, and finally, sixth columns represent average percentage improvements comparing with each scheme, respectively.

Figure 9 illustrates the performance of the proposed WGWO scheme for the energy consumption parameter as compared with existing schemes. After examining the graph, it is clear that the proposed approach is having a lower energy consumption than the existing schemes.

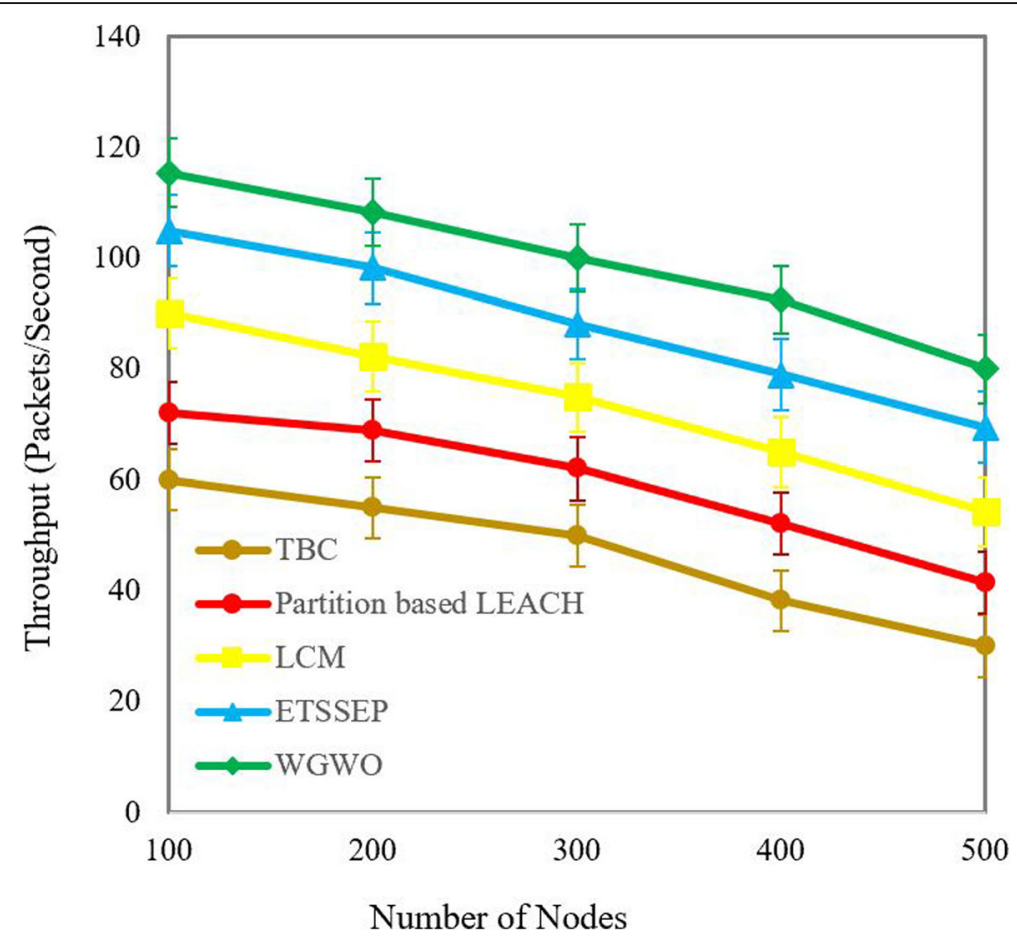

Fig. 7 Throughput (packets/second) vs. variation in the number of nodes 


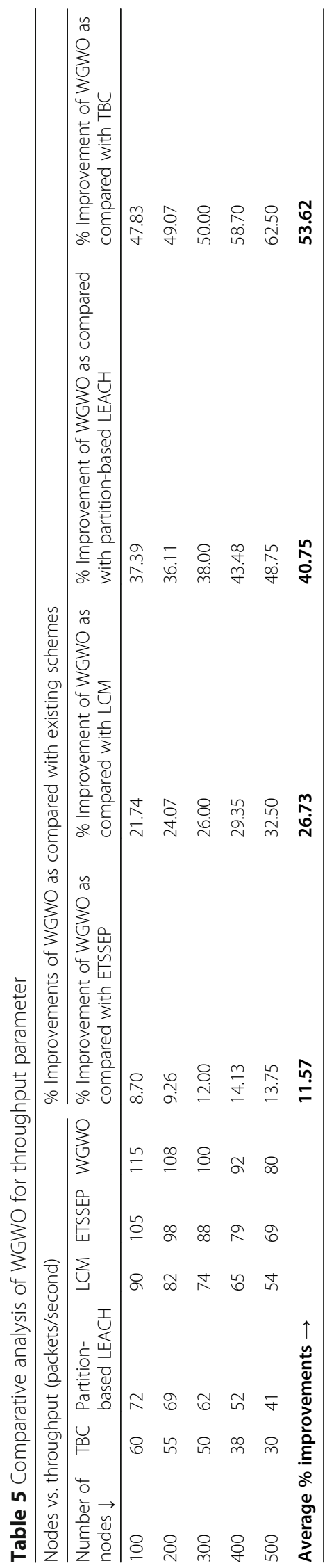




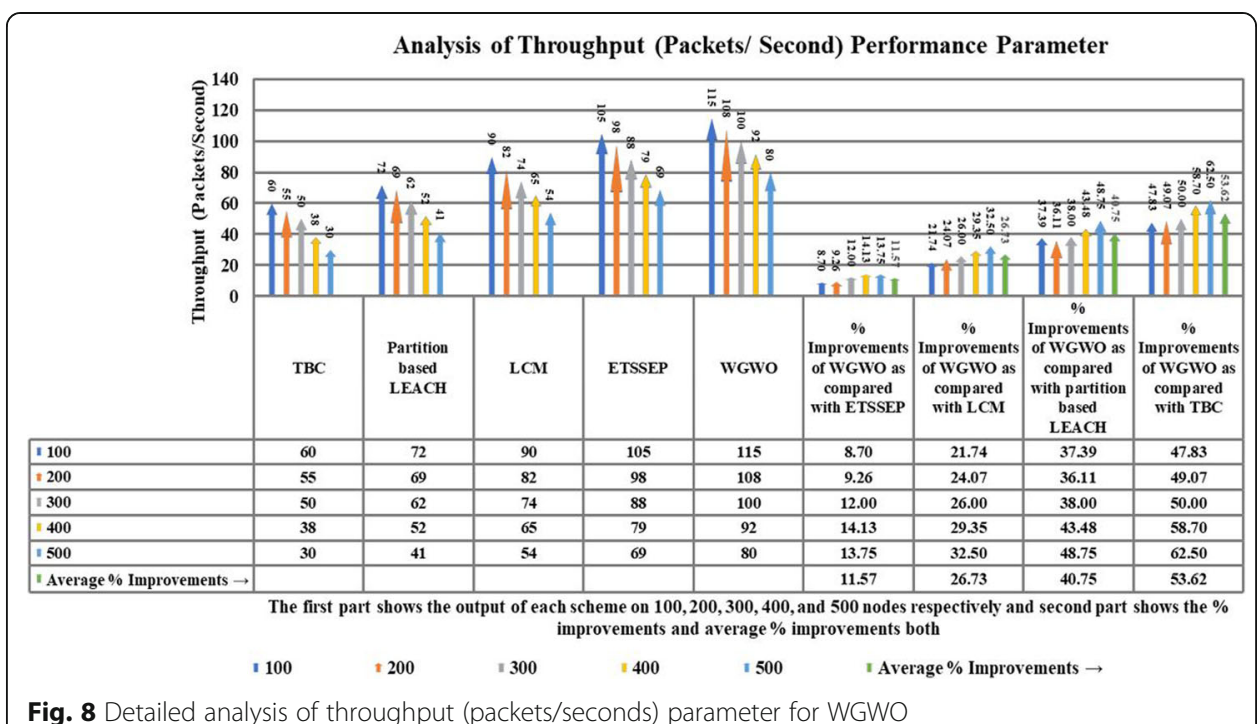

It can be stated from the above figure that the energy consumption parameter of our proposed scheme is lower. The energy consumption increases with the formation of non-optimal clusters. And, in the proposed research, with the help of a hybrid metaheuristic algorithm, the optimal number of clusters is formed with the objective of the minimum energy consumption. Further, in our proposed approach, communication as well as computing cost is low with optimal nodes selection as the next forwarders. Therefore, the proposed scheme achieves minimum energy consumption as compared with existing schemes. Next, Table 6 shows the comparative analysis of WGWO for the energy consumption parameter.

The average percentage improvements of WGWO in terms of energy consumption are $33.84,69.99,80.70$, and 88.56 as compared to ETSSEP, LCM, partition-based $\mathrm{LEACH}$, and TBC, respectively. Figure 10 depicts the detailed analysis of the energy consumption parameter.

Furthermore, Fig. 10 consists of five different columns. Initially, these five different columns represent the value of energy consumption parameter on 100, 200, 300, 400, and 500 nodes, and later in this figure, these five different columns represent the percentage improvements, and finally, sixth columns represent average percentage improvements comparing with each scheme, respectively.

Figure 11 indicates the performance of the proposed WGWO scheme for the network lifetime parameter as compared with existing schemes. After assessing the graph, it is clear that the proposed approach is having a higher network lifetime than the existing schemes.

From Fig. 11, it is evident that the network lifetime parameter of our proposed scheme is higher. The network lifetime is inversely proportional to the energy consumption parameter. The energy consumption is less in our proposed approach; consequently, network lifetime is higher. Further, in the existing schemes, routing decisions are made periodically without utilizing the impact of network conditions. In our proposed scheme, the routing decision is based on the characteristics of the next 


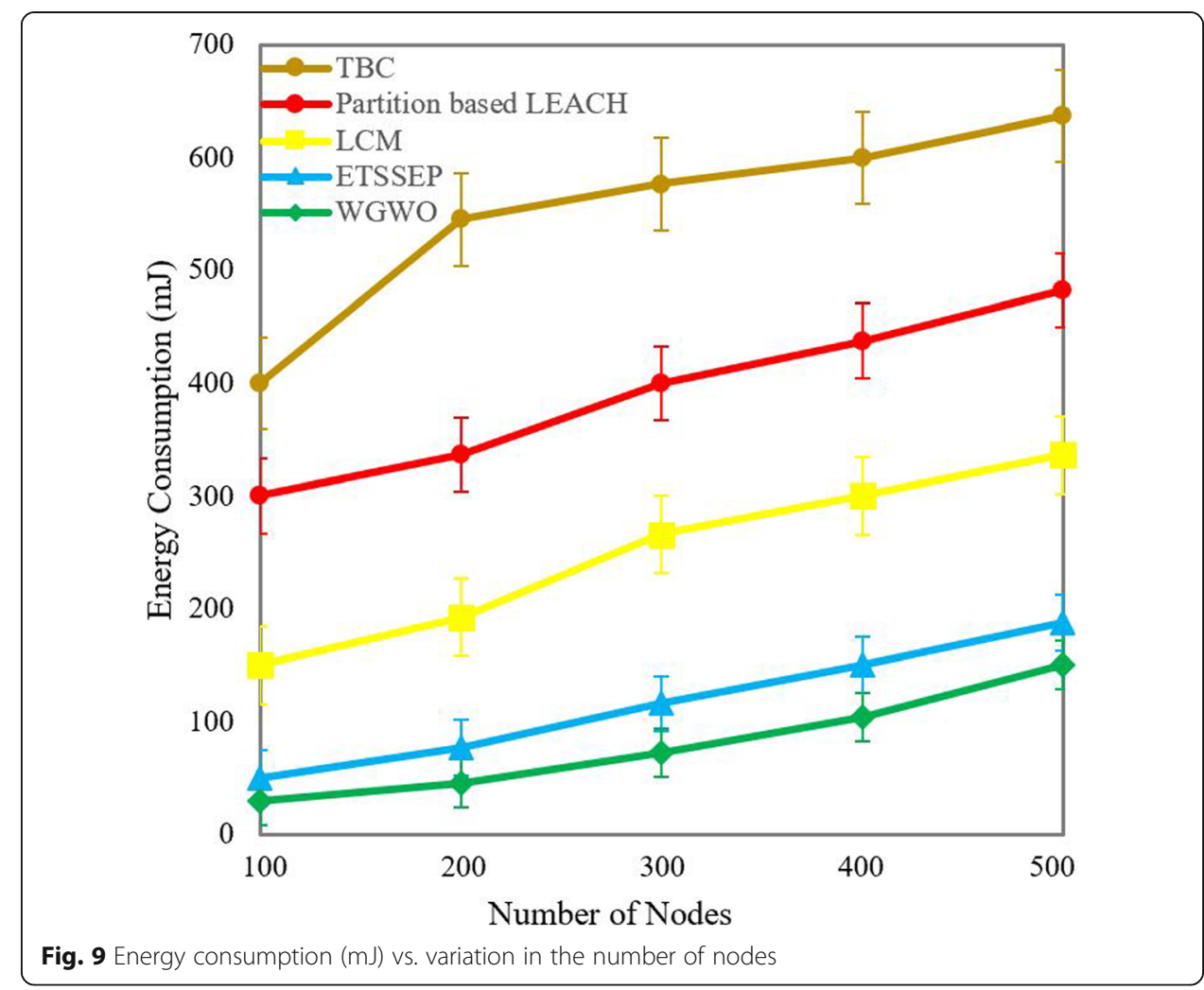

forwarders by utilizing energy-efficient criteria. And, this form the consistent routing mechanism. Next, Table 7 shows the comparative analysis of WGWO for the network lifetime parameter.

The average percentage improvements of WGWO in terms of network lifetime are 13.29, 31.29, 46.37, and 59.81 as compared to ETSSEP, LCM, partition-based LEACH, and TBC, respectively. Detailed analysis of the network lifetime parameter for WGWO is depicted in Fig. 12 which is in 10-point scale.

Furthermore, Fig. 12 consists of five different columns. Initially, these five different columns represent the value of network lifetime parameter on 100, 200, 300,400, and 500 nodes, and later in this figure these five different columns represent the percentage improvements, and finally, sixth columns represent average percentage improvements comparing with each scheme, respectively.

\section{Conclusion}

We have proposed a hybrid whale grey wolf optimization algorithm for novel energyefficient clustering for energy harvesting wireless sensor networks. The novel energyefficient clustering process consists of the optimal number of clusters formation and cluster heads $(\mathrm{CHs})$ selection in a dynamic fashion. Further, relay nodes are selected with the objective of energy efficiency, and later they are prioritized. The energyefficient clustering is done by the proposed hybrid WGWO algorithm. We use the various objective functions to compute the optimal number of clusters as well as selecting the $\mathrm{CH}$ node among multiple nodes in the cluster in a dynamic fashion. The hybrid WGWO algorithm can find better solutions for the fitness function with multiobjective functions. The multiple parameters are used to select the relay nodes to reach the 


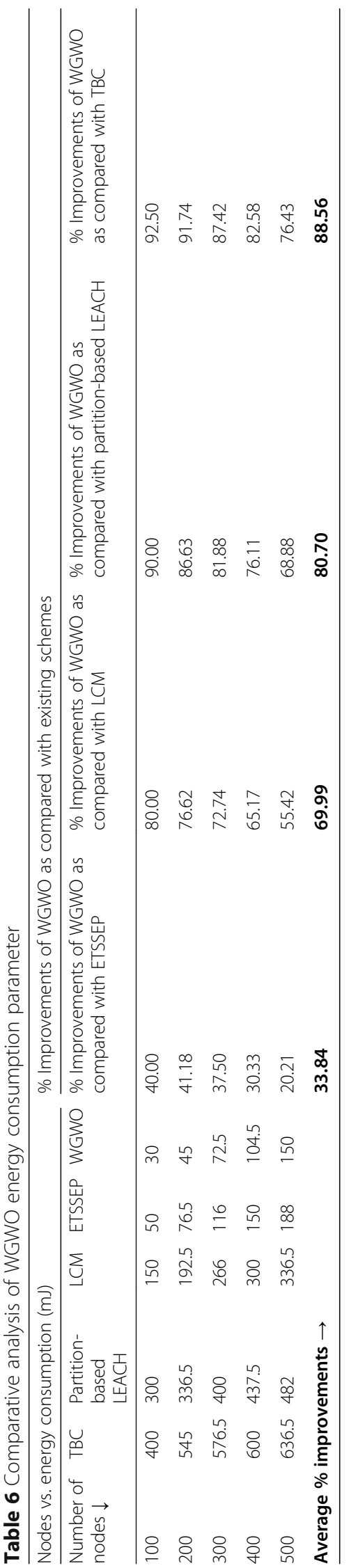




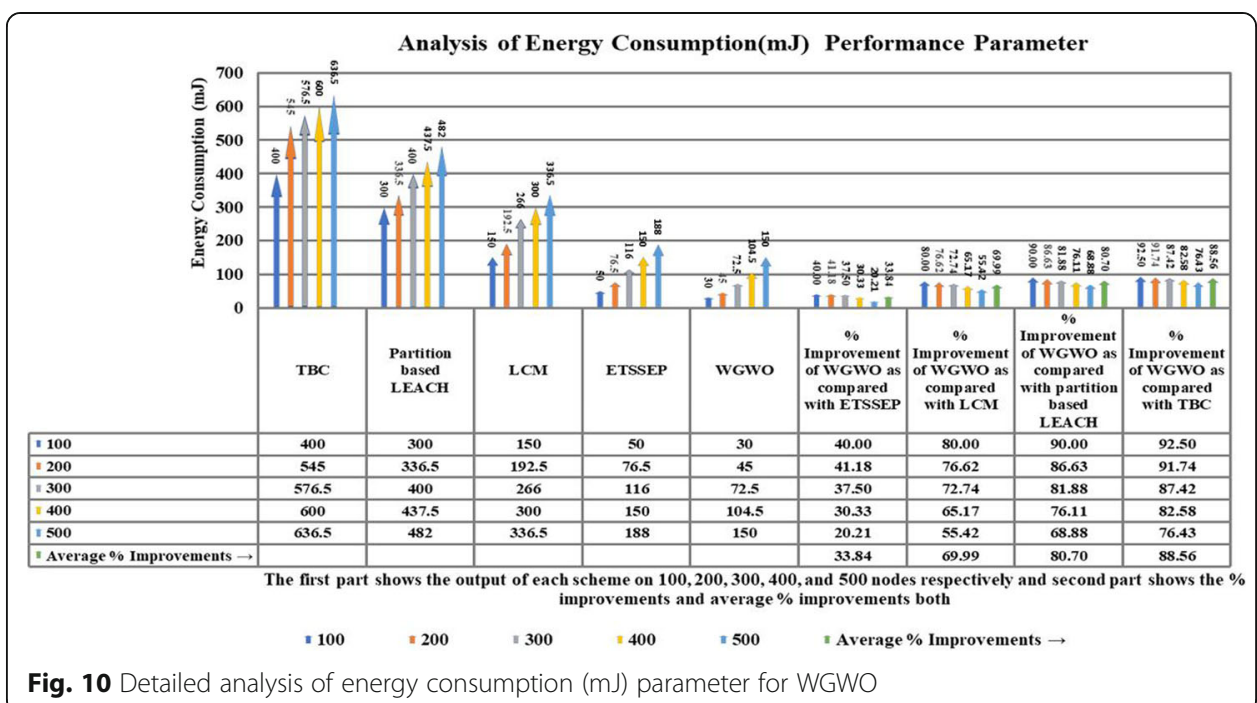

destination, and later these relay nodes are prioritized. Finally, the performance of the proposed WGWO protocol is compared with the existing ETSSEP, LCM, partition-based $\mathrm{LEACH}$, and TBC, respectively, in terms of delay, energy consumption, network lifetime, throughput, and delivery ratio. The results attest that the efficiency of the hybrid WGWO based clustering scheme is much better than the various existing schemes.

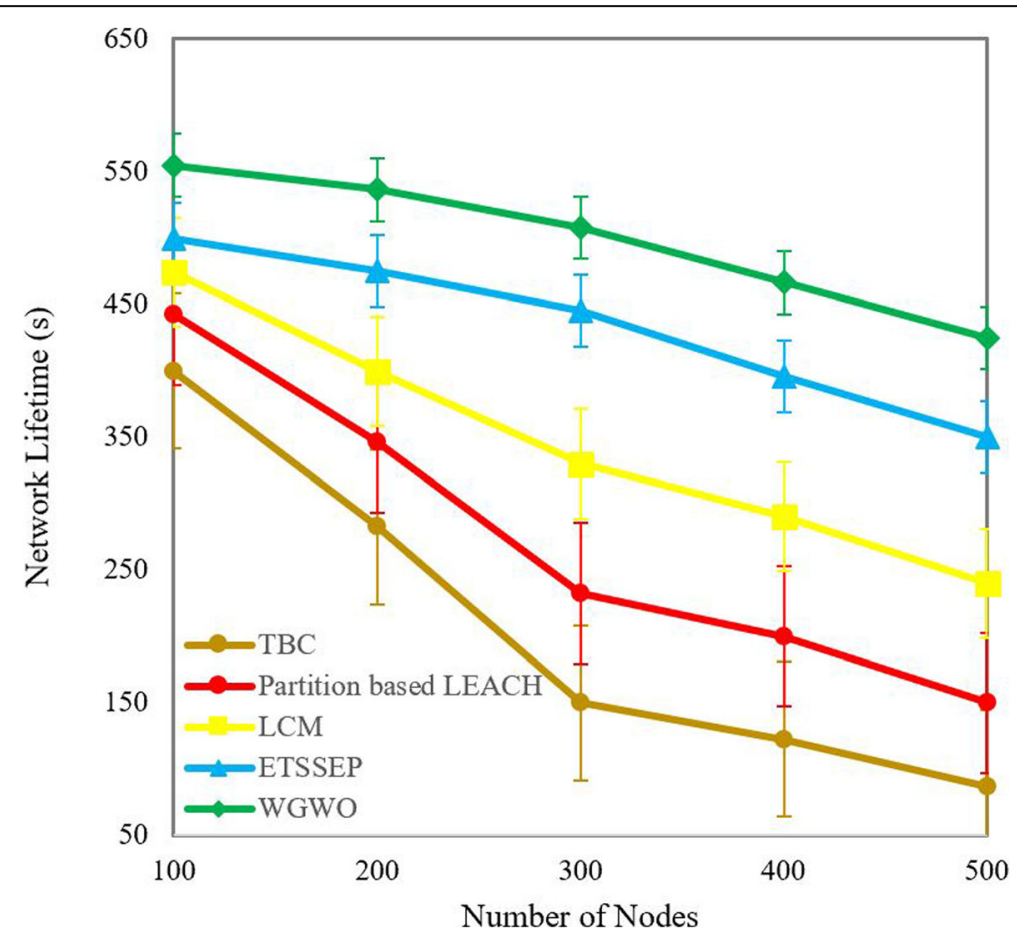

Fig. 11 Network lifetime(s) vs. variation in the number of nodes 







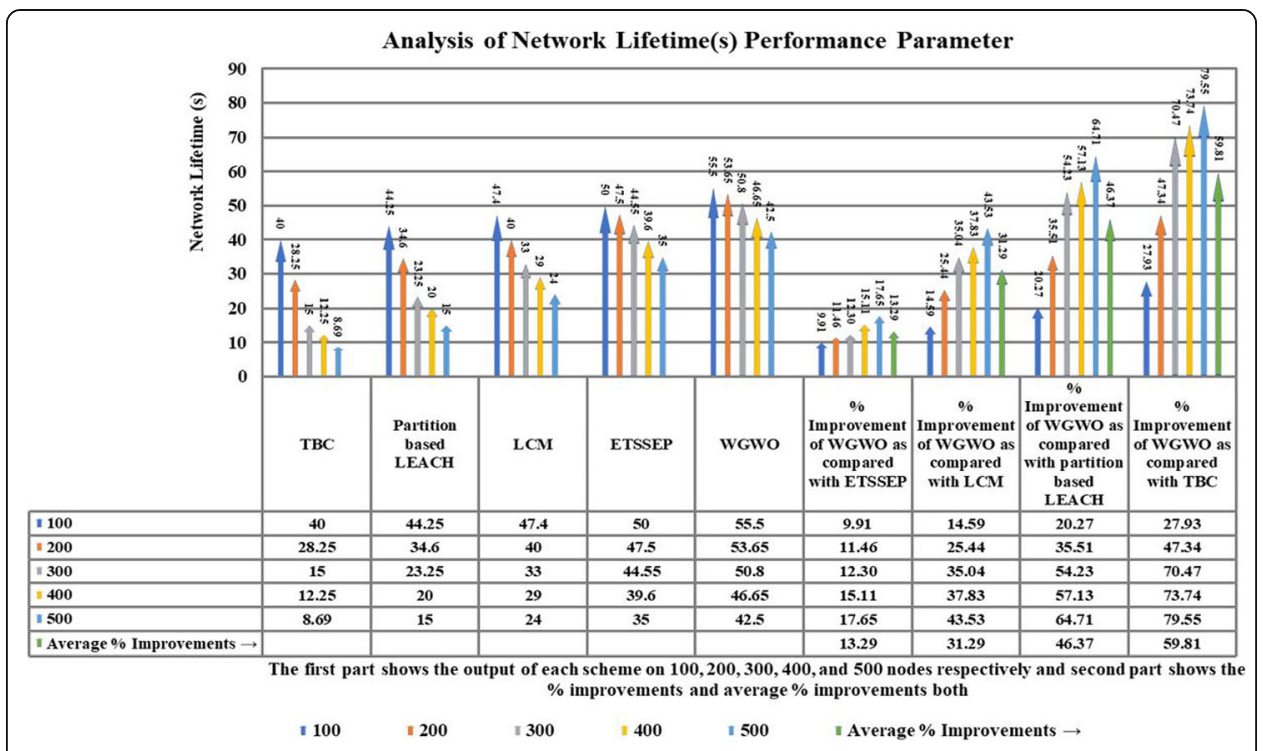

Fig. 12 Detailed analysis of network lifetime(s) parameter for WGWO

\section{Abbreviations}

WSNs: Wireless sensor networks; EH-WSNs: Energy harvesting wireless sensor networks; SIF: Swarm intelligence-based forwarding; CH: Cluster head; SN: Sensor nodes; OR: Opportunistic routing; LEACH: Low-energy adaptive clustering hierarchy; BS: Base station; ETSSEP: Enhanced threshold sensitive stable election protocol; FLDEAR: Fuzzy-logic, distance, and energy-aware routing; TBC: Tree-based clustering; LCM: Link-aware clustering mechanism; WOA: Whale optimization algorithm; GWO: Grey wolf optimization; WGWO: Whale grey wolf optimization; EDCW: Expected number of duty cycle wake-ups; EHPRP: EHWSNs Physuram inspired routing protocol; EOTC: Expected operating transmission cost; DC: Duty cycle; MAC: Medium access control

\section{Authors' contributions}

RSR has modeled and executed the research. SS and RK have supervised the research. RSR has designed experimental testing and data visualization. SP has validated mathematical design and testing. KA has enhanced the quality of the research by their valuable comments and suggestions in data analysis and discussion. YC has improved the technical writing of the paper with comments and suggestions. The authors read and approved the final manuscript.

\section{Funding}

The research is funded by the Manchester Metropolitan University, Manchester, UK, and supported by Deenbandhu Chhotu Ram University of Science and Technology, Murthal (Sonepat), Haryana, India.

\section{Availability of data and materials}

The experimental data and associated settings will be made available to researchers and practitioners on individual requests with the restrictions that it will be used only for further research in literature progress.

\section{Competing interest}

It is declared that there is no competing interest among authors.

\section{Author details}

${ }^{1}$ Department of Computer Science and Engineering, Deenbandhu Chhotu Ram University of Science and Technology, Murthal (Sonepat), Haryana 131039, India. ${ }^{2}$ Dr. A.P.J. Abdul Kalam Technical University, Lucknow, India. ${ }^{3}$ School of Engineering, Newcastle University, Newcastle upon Tyne NE1 7RU, UK. ${ }^{4}$ Department of Computing and Mathematics, Faculty of Science and Engineering, Manchester Metropolitan University, Manchester M1 5GD, UK. ${ }^{5}$ Department: Computing and Communications, Lancaster University, Lancaster LA1 4WA, UK.

Received: 3 March 2020 Accepted: 23 April 2020

Published online: 14 May 2020

\section{References}

1. O. Kaiwartya, A.H. Abdullah, Y. Cao, J. Lloret, S. Kumar, R.R. Shah, M. Prasad, S. Prakash, Virtualization in wireless sensor networks: fault tolerant embedding for internet of things. IEEE Internet of Things Journal 5(2), $571-580$ (2017)

2. Ullah, F., Abdullah, A.H., Kaiwartya, O. and Cao, Y., 2017. TraPy-MAC: Traffic priority aware medium access control protocol for wireless body area network. Journal of medical systems, $41(6)$, p.93. 
3. Qureshi, K.N., Abdullah, A.H., Kaiwartya, O., Ullah, F., labal, S. and Altameem, A., 2016. Weighted link quality and forward progress coupled with modified RTS/CTS for beaconless packet forwarding protocol (B-PFP) in VANETs. Telecommunication Systems, pp.1-16.

4. Khatri A, Kumar, S., Kaiwartya, O. and Abdullah, A.H., 2017. Green computing for wireless sensor networks: optimization and Huffman coding approach. Peer-to-Peer Networking and Applications, 10(3), pp.592-609.

5. J. Niu, L. Cheng, Y. Gu, L. Shu, S.K. Das, R3E: Reliable reactive routing enhancement for wireless sensor networks. IEEE Transactions on Industrial Informatics 10(1), 784-794 (2013)

6. D. Zhang, G. Li, K. Zheng, X. Ming, Z.H. Pan, An energy-balanced routing method based on forward-aware factor for wireless sensor networks. IEEE transactions on industrial informatics 10(1), 766-773 (2013)

7. C. Yang, K.W. Chin, On nodes placement in energy harvesting wireless sensor networks for coverage and connectivity. IEEE Transactions on Industrial Informatics 13(1), 27-36 (2016)

8. S. Manfredi, E. Di Tucci, Decentralized control algorithm for fast monitoring and efficient energy consumption in energy harvesting wireless sensor networks. IEEE Transactions on Industrial Informatics 13(4), 1513-1520 (2016)

9. M.S. Bahbahani, E. Alsusa, A cooperative clustering protocol with duty cycling for energy harvesting enabled wireless sensor networks. IEEE Transactions on Wireless Communications 17(1), 101-111 (2017)

10. C. Wang, J. Li, Y. Yang, F. Ye, Combining solar energy harvesting with wireless charging for hybrid wireless sensor networks. IEEE Transactions on Mobile Computing 17(3), 560-576 (2017)

11. C. Wang, S. Guo, Y. Yang, An optimization framework for mobile data collection in energy-harvesting wireless sensor networks. IEEE Transactions on Mobile Computing 15(12), 2969-2986 (2016)

12. L. Cheng, J. Niu, J. Cao, S.K. Das, Y. Gu, QoS aware geographic opportunistic routing in wireless sensor networks. IEEE Transactions on Parallel and Distributed Systems 25(7), 1864-1875 (2013)

13. T. Lu, G. Liu, S. Chang, Energy-efficient data sensing and routing in unreliable energy-harvesting wireless sensor network. Wireless Networks 24(2), 611-625 (2018)

14. M.H. Anisi, G. Abdul-Salaam, M.Y.I. Idris, A.W.A. Wahab, I. Ahmedy, Energy harvesting and battery power based routing in wireless sensor networks. Wireless Networks 23(1), 249-266 (2017)

15. L.A. Villas, A. Boukerche, H.S. Ramos, H.A.F. De Oliveira, R.B. de Araujo, A.A.F. Loureiro, DRINA: A lightweight and reliable routing approach for in-network aggregation in wireless sensor networks. IEEE Transactions on Computers 62(4), 676-689 (2012)

16. Y. Liu, K.Y. Lam, S. Han, Q. Chen, Mobile data gathering and energy harvesting in rechargeable wireless sensor networks. Information Sciences 482, 189-209 (2019)

17. S. Sarang, M. Drieberg, A. Awang, R. Ahmad, A QOS MAC protocol for prioritized data in energy harvesting wireless sensor networks. Computer networks 144, 141-153 (2018)

18. S.M. Bozorgi, A.S. Rostami, A.A.R. Hosseinabadi, V.E. Balas, A new clustering protocol for energy harvesting-wireless sensor networks. Computers \& Electrical Engineering 64, 233-247 (2017)

19. Y. Wu, W. Liu, Routing protocol based on genetic algorithm for energy harvesting-wireless sensor networks. IET Wireless Sensor Systems 3(2), 112-118 (2013)

20. C. Petrioli, M. Nati, P. Casari, M. Zorzi, S. Basagni, ALBA-R: load-balancing geographic routing around connectivity holes in wireless sensor networks. IEEE Transactions on Parallel and Distributed Systems 25(3), 529-539 (2013)

21. Kumar, S., Verma, S.K. and Kumar, A., 2015. Enhanced threshold sensitive stable election protocol for heterogeneous wireless sensor network. wireless personal communications, 85(4), pp.2643-2656.

22. S.S. Wang, Z.P. Chen, LCM: A link-aware clustering mechanism for energy-efficient routing in wireless sensor networks. IEEE sensors journal 13(2), 728-736 (2012)

23. Kim, K.T., Lyu, C.H., Moon, S.S. and Youn, H.Y., 2010, April. Tree-based clustering (TBC) for energy efficient wireless sensor networks. In 2010 IEEE 24th International Conference on Advanced Information Networking and Applications Workshops (pp. 680-685). IEEE.

24. Gou, H., Yoo, Y. and Zeng, H., 2009, October. A partition-based LEACH algorithm for wireless sensor networks. In 2009 Ninth IEEE International Conference on Computer and Information Technology (Vol. 2, pp. 40-45). IEEE.

25. Z.M. Zahedi, R. Akbari, M. Shokouhifar, F. Safaei, A. Jalali, Swarm intelligence based fuzzy routing protocol for clustered wireless sensor networks. Expert Systems with Applications 55, 313-328 (2016)

26. W. Zhang, G. Han, Y. Feng, J. Lloret, IRPL: An energy efficient routing protocol for wireless sensor networks. Journal of Systems Architecture 75, 35-49 (2017)

27. K.A. Darabkh, N.J. Al-Maaitah, I.F. Jafar, K. Ala'F, EA-CRP: a novel energy-aware clustering and routing protocol in wireless sensor networks. Computers \& Electrical Engineering 72, 702-718 (2018)

28. R.E. Mohemed, A.I. Saleh, M. Abdelrazzak, A.S. Samra, Energy-efficient routing protocols for solving energy hole problem in wireless sensor networks. Computer Networks 114, 51-66 (2017)

29. R.E. Mohamed, W.R. Ghanem, A.T. Khalil, M. Elhoseny, M. Sajjad, M.A. Mohamed, Energy efficient collaborative proactive routing protocol for wireless sensor network. Computer Networks 142, 154-167 (2018)

30. A.E. Fawzy, M. Shokair, W. Saad, Balanced and energy-efficient multi-hop techniques for routing in wireless sensor networks. IET Networks 7(1), 33-43 (2017)

31. H. Huang, H. Yin, G. Min, J. Zhang, Y. Wu, X. Zhang, Energy-aware dual-path geographic routing to bypass routing holes in wireless sensor networks. IEEE Transactions on Mobile Computing 17(6), 1339-1352 (2017)

32. H. Rhim, K. Tamine, R. Abassi, D. Sauveron, S. Guemara, A multi-hop graph-based approach for an energy-efficient routing protocol in wireless sensor networks. Human-centric Computing and Information Sciences 8(1), 1-21 (2018)

33. A. Hawbani, X. Wang, A. Abudukelimu, H. Kuhlani, Y. Al-sharabi, A. Qarariyah, A. Ghannami, Zone probabilistic routing for wireless sensor networks. IEEE Transactions on Mobile Computing 18(3), 728-741 (2018)

34. F. Li, M. Xiong, L. Wang, H. Peng, J. Hua, X. Liu, A novel energy-balanced routing algorithm in energy harvesting sensor networks. Physical Communication 27, 181-187 (2018)

35. A. Hawbani, X. Wang, Y. Sharabi, A. Ghannami, H. Kuhlani, S. Karmoshi, LORA: load-balanced opportunistic routing for asynchronous duty-cycled WSN. IEEE Transactions on Mobile Computing 18(7), 1601-1615 (2018)

36. Z. Mottaghinia, A. Ghaffari, Fuzzy logic based distance and energy-aware routing protocol in delay-tolerant mobile sensor networks. Wireless Personal Communications 100(3), 957-976 (2018) 
37. W. Tang, K. Zhang, D. Jiang, Physarum-inspired routing protocol for energy harvesting wireless sensor networks. Telecommunication Systems 67(4), 745-762 (2018)

38. Mood, S.E. and Javidi, M.M., 2019. Energy-efficient clustering method for wireless sensor networks using modified gravitational search algorithm. Evolving Systems, pp.1-13.

39. A. Barzin, A. Sadeghieh, H. Khademi Zareh, M. Honarvar, Hybrid swarm intelligence-based clustering algorithm for energy management in wireless sensor networks. Journal of Industrial and Systems Engineering 12(3), 78-106 (2019)

40. S. Mirjalili, A. Lewis, The whale optimization algorithm. Advances in engineering software 95, 51-67 (2016)

41. S. Mirjalili, S.M. Mirjalili, A. Lewis, Grey wolf optimizer. Advances in engineering software 69, 46-61 (2014)

42. X. Liu, J. Wu, A method for energy balance and data transmission optimal routing in wireless sensor networks. Sensors 19(13), 3017 (2019)

43. Khasawneh, A., Latiff, M.S.B.A., Kaiwartya, O. and Chizari, H., 2017. Next forwarding node selection in underwater wireless sensor networks (UWSNs): techniques and challenges. Information, 8(1), p.3.

44. Ganesan, S.; Ramaswamy, k.; Basha, A.; M, H. NOSS (A Novel Optimal Sleep Scheduling) scheme for energy and data reliability optimization in mobile cloud assisted wireless sensor networks. J. Eng. 2019.

45. K.N. Qureshi, A.H. Abdullah, O. Kaiwartya, S. lqbal, R.A. Butt, F. Bashir, A dynamic congestion control scheme for safety applications in vehicular ad hoc networks. Computers \& Electrical Engineering 72, 774-788 (2018)

46. E. Ghadimi, O. Landsiedel, P. Soldati, S. Duquennoy, M. Johansson, Opportunistic routing in low duty-cycle wireless sensor networks. ACM T. Sensor Network 10(4), 1-39 (2014)

47. X. Zhang, C. Wang, L. Tao, An opportunistic packet forwarding for energy-harvesting wireless sensor networks with dynamic and heterogeneous duty cycle. IEEE Sens. Lett. 2(3), 1-4 (2018)

\section{Publisher's Note}

Springer Nature remains neutral with regard to jurisdictional claims in published maps and institutional affiliations.

\section{Submit your manuscript to a SpringerOpen ${ }^{\circ}$} journal and benefit from:

- Convenient online submission

- Rigorous peer review

- Open access: articles freely available online

High visibility within the field

- Retaining the copyright to your article

Submit your next manuscript at $\boldsymbol{\nabla}$ springeropen.com 